\title{
Flagella, flexibility and flow: Physical processes in microbial ecology
}

\author{
D.R. Brumley ${ }^{1,3}$, R. Rusconi ${ }^{1,3}$, K. Son ${ }^{1,2}$, and R. Stocker ${ }^{1,3, a}$ \\ 1 Ralph M. Parsons Laboratory, Department of Civil and Environmental Engineering, \\ Massachusetts Institute of Technology, Cambridge MA 02139, USA \\ 2 Department of Mechanical Engineering, Massachusetts Institute of Technology, \\ Cambridge MA 02139, USA \\ 3 Department of Civil, Environmental and Geomatic Engineering, ETH Zurich, 8093 \\ Zurich, Switzerland
}

Received 25 June 2015 / Received in final form 2 November 2015

Published online 15 December 2015

\begin{abstract}
How microorganisms interact with their environment and with their conspecifics depends strongly on their mechanical properties, on the hydrodynamic signatures they generate while swimming and on fluid flows in their environment. The rich fluid-structure interaction between flagella - the appendages microorganisms use for propulsion - and the surrounding flow, has broad reaching effects for both eukaryotic and prokaryotic microorganisms. Here, we discuss selected recent advances in our understanding of the physical ecology of microorganisms, which have hinged on the ability to directly interrogate the movement of individual cells and their swimming appendages, in precisely controlled fluid environments, and to image them at appropriately fast timescales. We review how a flagellar buckling instability can unexpectedly serve a fundamental function in the motility of bacteria, we elucidate the role of hydrodynamics and flexibility in the emergent properties of groups of eukaryotic flagella, and we show how fluid flows characteristic of microbial habitats can strongly bias the migration and spatial distribution of bacteria. The topics covered here are illustrative of the potential inherent in the adoption of experimental methods and conceptual frameworks from physics in understanding the lives of microorganisms.
\end{abstract}

\section{Introduction}

Fluid mechanics at the microscale governs a myriad of physical, chemical and biological processes, from the self-propulsion of individual microorganisms [1] and their collective motions [2], to the transport of solutes [3], the interaction between cells [4], and the formation of biofilms [5]. Despite occurring at the microscale, these processes have profound macroscale consequences across a broad range of organisms. Cilia-driven flows establish the left-right asymmetry in developing embryos [6] and

\footnotetext{
${ }^{a}$ e-mail: romanstocker@ethz.ch
} 
significantly enhance nutrient exchange between corals and their environment [7], while environmental flows can influence the spatial distribution of microorganisms [8] and affect the development of biofilms [9].

Microscale fluid flows interacting with flexible structures are ubiquitous in living systems. Flexibility allows red blood cells to squeeze through capillaries [10] and DNA to change shape in response to specific binding requirements [11]. Flagella and cilia the propulsion appendages used by microorganisms - often operate by exploiting mechanical instabilities. The coupling between these flexible structures and the surrounding fluid flow can give rise to rich dynamics, from the "run-reverse-flick" motion of monotrichous bacteria [12], to collective beating of ciliary arrays [13]. Additionally, shear flows characteristic of microbial habitats can lead to the passive aggregation of microorganisms in specific regions of a flowing fluid [14].

For a fluid with dynamic viscosity $\mu$, density $\rho$, characteristic velocity $u$ and length scale $l$, the Reynolds number of the flow, $\operatorname{Re}=\rho u l / \mu$, is a dimensionless number quantifying the relative magnitude of inertial forces and viscous forces. Owing to their small size $[1-100 \mu \mathrm{m}]$ and typical velocities $[1-100 \mu \mathrm{m} / \mathrm{s}]$, the various organisms we shall discuss in this review all operate in an essentially inertialess world $\left[10^{-6}<\right.$ $\operatorname{Re}<10^{-2}$ ] in which fluid motions are governed by the overdamped, time-reversible Stokes equations:

$$
\nabla p=\mu \nabla^{2} \mathbf{u}, \quad \nabla \cdot \mathbf{u}=0,
$$

where $\mathbf{u}$ and $p$ are the velocity and pressure of the fluid respectively. The flagella of eukaryotic and prokaryotic organisms must exhibit nonreciprocal motions in order to break the temporal symmetry of these equations and achieve locomotion [15]. In this context, flexibility (or elasticity) is particularly important, as it provides an additional means of breaking the time-reversibility of the Stokes equations and achieving propulsion. Under these conditions, a time-reversible actuation - such as the continuous switching in direction of a bacterial flagellar motor - can give rise to a non-reciprocal motion when combined with flexibility. The inherent flexibility of microbial appendages can have a direct influence on their overall motility and collective dynamics. Despite the structural differences between eukaryotic and prokaryotic flagella, recent work highlights that fluid flow and flexibility are inextricably linked in the ecology of both classes of microorganisms, and we therefore include both in this discussion.

In this paper, we investigate the effects of flow and flexibility on the physical ecology of microorganisms. In particular, we illustrate several specific examples in which the ability to deconstruct complex processes into constituent parts, confine and control the processes under investigation in well-defined microenvironments, and directly visualise them using high-speed imaging, has yielded new insights into the physical ecology of microorganisms. We focus our attention on examples in which the subtle interplay between flows - either organism-generated or externally imposed and microbial mechanics, can have profound effects on the overall motility patterns and collective dynamics.

\section{Flexibility in bacterial flagellar dynamics}

Bacteria use rigid helical flagella for propulsion, often rotating them like a corkscrew to achieve locomotion [16]. The prokaryotic flagellum is composed of three main structural elements - the basal body, the hook, and the flagellar filament [17]. The basal body is embedded in the cell membrane and consists of multiple rings and a rod that is connected to a proton-driven or sodium-driven rotary motor for actuation. Just 
outside the cell membrane, a 50-100 nm long and $\sim 20 \mathrm{~nm}$ wide "hook" connects the rigid helical flagellar filament to the basal body. The helicity and the rotational direction of the flagellum dictate the cell's swimming modes and directions. The ability of cells to reorient themselves is an essential part of their survival strategy leading to chemotaxis [18], which is the ability of cells to sense external chemical gradients and to bias their motility towards favourable environmental conditions.

The best studied organism for bacterial locomotion is the enteric bacterium Escherichia coli $[19,20]$. E. coli has multiple (4-10) flagellar filaments emerging from random locations on its cell body (Fig. 1A). Each flagellum is powered by a proton motor, and when all flagella rotate counter-clockwise (as seen from behind), the flagella form a bundle that propels the cell forward in a nearly straight "run". When one or more motors change their direction of rotation, the bundle comes apart and the resulting torque from the flagella unbundling process causes the cell to reorient, or "tumble", in a nearly random direction (Fig. 1B). Especially during the unbundling process, when a rapid directional change in the motor produces a twisting torque, the flagellar filaments undergo polymorphic transformations [21,22] caused by cooperative conformational changes of a biological polymer called flagellin. This causes the handedness of the helix to change from the normal left-handed shape during the "run", which has been computationally shown to be most efficient for propulsion among all observed polymorphisms [23], to the semi-coiled and curly right-handed shapes during the "tumble".

The picture of bacterial locomotion just described is independent of flexibility, and models of bacterial locomotion routinely assumed that flagella are rigid. However, recent studies have revealed that flexibility serves important functions in prokaryotic flagella, for both enteric and marine bacteria. For E. coli, Brown and coworkers experimentally verified that the hook's flexibility is essential for the flagellar bundle formation, by changing the bending stiffness of the hook through genetic engineering [24]. They found that cells with stiffer hooks show an atypical swimming behaviour as a consequence of disrupted bundle formation [24]. Even more profound is the role of the hook's flexibility in marine bacteria, which we focus on in what follows.

The morphology and swimming strategies of bacteria living in different habitats from $E$. coli can be radically different from the strategies of this important model bacterium. The vast majority $(90 \%)$ of motile bacterial species in the ocean have only a single flagellum [25] (Fig. 1C) and they were recently discovered to execute a cyclic three-step motility pattern, "run-reverse-flick", that is radically different from E. coli's run-and-tumble [26]. E. coli cells are "pushers", as they always swim forwards with their flagella at the rear. However, single-flagellated bacteria alternate between the "pusher" mode and the "puller" mode (swimming backwards when flagella pull the cell) by changing the rotational direction of their one flagellar filament. Xie and coworkers discovered that the transition from a forward run to a backward run always results in a $180^{\circ}$ change in swimming direction, which is a "reversal" [26] rather than a U-turn. However, in the opposite transition from a backward run to a forward run, the flagellum of the marine bacterium Vibrio alginolyticus undergoes a large, off-axis deformation called a "flick", which allows cells to reorient by an average angle of $90^{\circ}$ (Fig. 1D). Since the flagellum of $V$. alginolyticus is covered in a sheath, polymorphic transformations are not possible, pointing at a different mechanism underpinning the flicks.

The mechanism responsible for the flicks has been elucidated by Son and coworkers [12], who captured this reorientation process using high-speed observations at up to 1,000 frames per second (Fig. 1E). High-speed imaging revealed that the flick occurs $\sim 10 \mathrm{~ms}$ after the onset of a forward run, rather than at the end of a backward run, and this provided a mechanistic clue as to the flick's origin by enabling correct assessment of the hydrodynamic loads on the cell. During this $\sim 10 \mathrm{~ms}$ long forward 


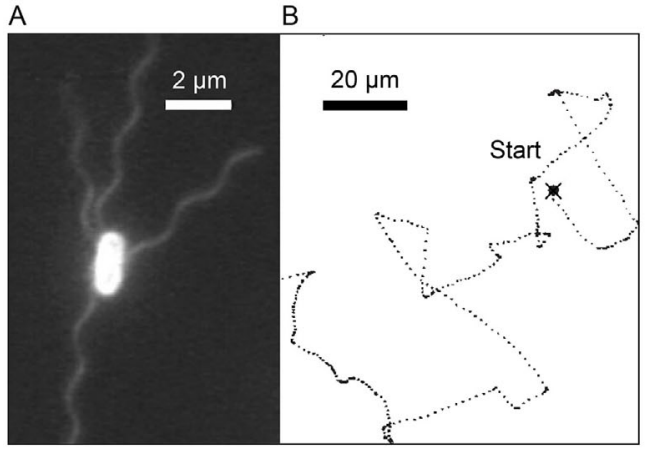

E

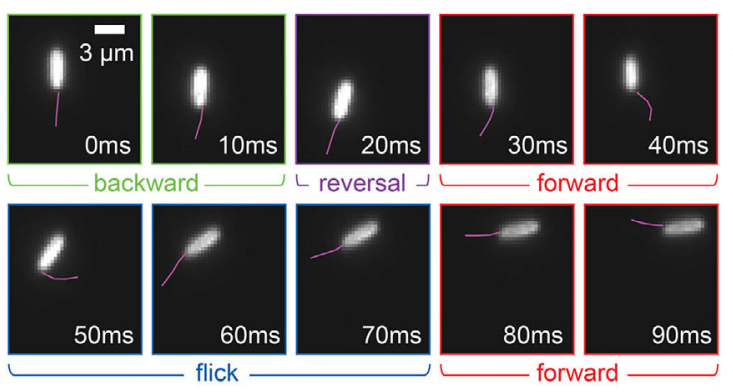

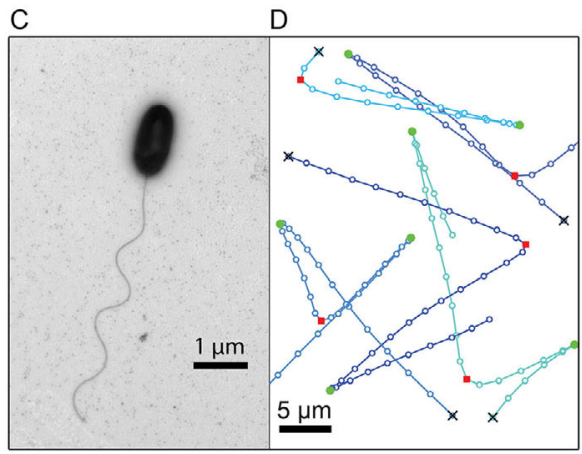

$\mathrm{F}$

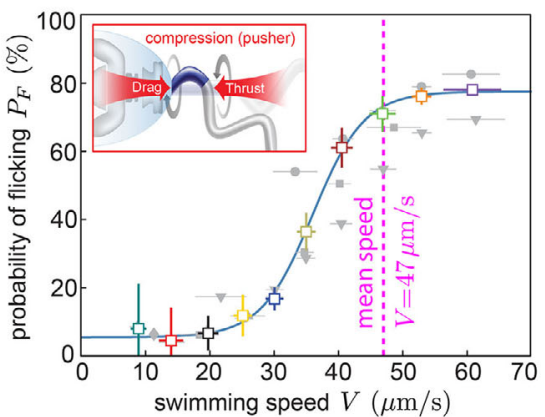

Fig. 1. (A) Fluorescent image of an immobilised Escherichia coli bacterium. Reproduced with permission from the American Society for Microbiology. Turner, L., Ryu, W.S., and Berg, H.C. (2000) Journal of Bacteriology 182(10), 2793-2801, doi: 10.1128/JB.182.10.27932801.2000 [21]. (B) A trajectory of an E. coli cell swimming in a run-and-tumble pattern captured at 12.6 frames/s. Reprinted by permission from Macmillan Publishers Ltd: Berg, H.C. and Brown, D.A. (1972) Nature 239(5374), 500-504, doi: 10.1038/239500a0 [18]. (C) Transmission electron micrograph of the monotrichous marine bacterium Vibrio alginolyticus. Reproduced with permission from the American Society for Microbiology. Stocker, R. and Seymour, J.R. (2012) Microbiology and Molecular Biology Reviews 76(4), 792-812, doi: 10.1128/MMBR.00029-12 [27]. (D) Run-reverse-flick motility in marine bacteria captured at 30 frames/s, showing $180^{\circ}$ reversals (green circles) and reorientations distributed around a mean of $90^{\circ}$ that are consistent with flicks (red squares) [12]. (E) Image sequence showing the kinematics of the flagellum (magenta) during a flick captured with high-intensity dark-field microscopy at 420 frames/s [12]. (F) The flick results from a buckling instability of the hook (inset). The axial and torsional loads on the hook are linearly proportional to the cell's swimming speed. The probability $P_{F}$ that a cell flicks during a swimming cycle increases sharply with swimming speed when speed exceeds a value $(\sim 35 \mu \mathrm{m} / \mathrm{s})$ consistent with the critical load for buckling. The blue curve is a logistic fit, and horizontal and vertical error bars denote standard deviations. Reprinted by permission from Son, K., Guasto, J.S., Stocker, R. (2013) Nature Physics 9(8), 494-498 doi: 10.1038/NPHYS2676 [12].

motion, the compressive forces exerted on the cell by the drag on the head and the thrust from the flagellum cause a mechanical buckling instability at the base of the flagellum, and in particular in the hook, which is known to be considerably more compliant than the flagellar filament. The mechanical origin of the flick was supported by experiments where the cell's swimming speed and thus the compressive force on the hook was changed (by changing the sodium concentration in the medium), which resulted in a sharp decrease in flicking below a threshold speed that was consistent with the critical load predicted for buckling (Fig. 1F). 
A
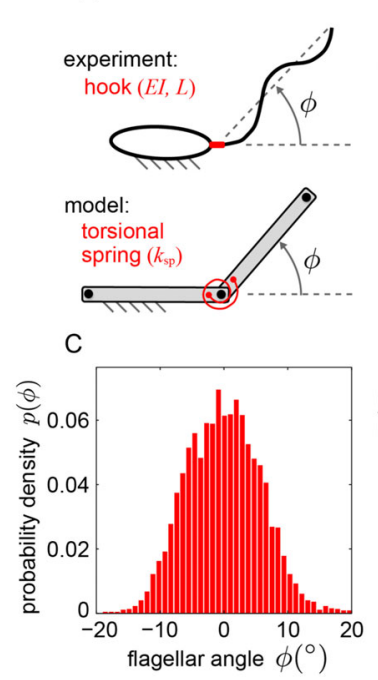
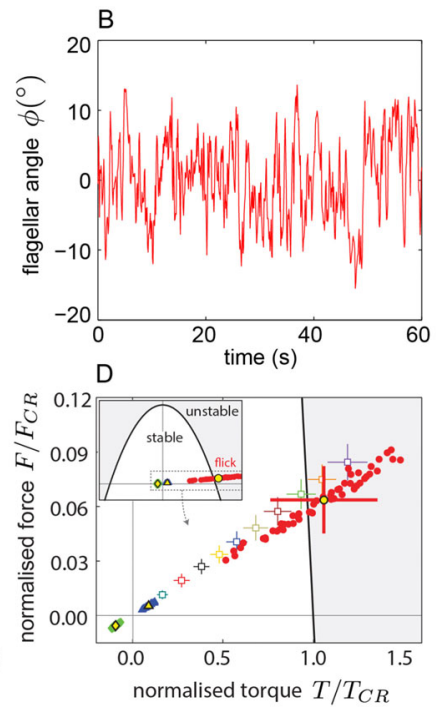

Fig. 2. The hook's bending stiffness is measured from the fluctuations in the flagellar orientation of cells that are immobilised on a glass slide. (A) The flagellar filament and the cell head are approximated as rigid links and the hook (red) as a torsional spring [12]. (B) Time series and (C) probability density of the flagellar orientation angle. (D) Stability diagram of the hook under combined axial and torsional loads. The hook is unstable when the normalised viscous force $\left(F / F_{C R}\right)$ and torque $\left(T / T_{C R}\right)$ fall outside the stability boundary (black curve). Critical loads were computed from the bending stiffness measurements (panels A-C). Reprinted by permission from Son, K., Guasto, J.S., Stocker, R. (2013) Nature Physics 9(8), 494-498, doi: 10.1038/NPHYS2676.

The hook's bending stiffness has been directly measured [12], and the bucklinginduced flicking can be understood in terms of the hook's stability diagram, obtained by approximating the hook as an Euler beam. The hook of $V$. alginolyticus is a straight, hollow, slender rod (slenderness ratio $=20$ ) subjected to a viscous force $(F)$ and torque $(T)$, which stem from the drag-based thrust of the cell [28]. The hook can buckle above a critical load under compression $F>F_{C R}=\pi^{2} E I / L^{2}$, under torsion $T>T_{C R}=2 \pi E I / L$, or under simultaneous loading when $F / F_{C R}+\left(T / T_{C R}\right)^{2}>$ 1 [29]. The critical loads on the hook were calculated by directly measuring the bending stiffness EI of the hook in the flicking state using non-motile $V$. alginolyticus cells with the cell head immobilised on a glass substrate and their flagellar filament undergoing Brownian fluctuations [12]. These fluctuations stem from the deformation of the flexible hook, which can be modelled as a torsional spring with spring constant $k_{s p} \approx E I / L$, connecting two rigid links that represent the cell head and the flagellar filament (Fig. 2A). Assuming the spring to be linear with the head-filament orientation angle $\phi[30,31]$, the elastic energy $U$ of the discrete torsional spring system is $U=k_{s p} \phi^{2} / 2$. The thermal energy of the surrounding medium drives the flagellar fluctuations and thus the probability $p(\phi)$ of occupying a given energy state with angle $\phi$ is given by the Boltzmann relation $p(\phi)=p_{0} \exp \left(-k_{s p} \phi^{2} / 2 k_{B} T_{A}\right)$, where $k_{B}$ is Boltzmann's constant, $T_{A}$ is the absolute temperature, and $p_{0}$ is a normalisation constant. The variance of the orientation angle, $\left\langle\phi^{2}\right\rangle$, is experimentally measurable (Fig. 2B-C) and is related to the spring constant, $k_{s p}$, through the variance of the Boltzmann distribution by $\left\langle\phi^{2}\right\rangle=k_{B} T_{A} / k_{s p}$, which yields an expression for the bending stiffness,

$$
E I=L \frac{k_{B} T_{A}}{\left\langle\phi^{2}\right\rangle} .
$$


At the instant of flicking, the normalised force and torque exceed the marginal stability condition, $F / F_{C R}+\left(T / T_{C R}\right)^{2}=1$, and the hook buckles (Fig. 2D). Based on observations of trajectories of other cultured marine isolates, as well as natural marine communities of bacteria, this reorientation mechanism appears to be widespread and possibly preponderant among marine bacteria, and it represents one of the smallest examples in nature of controlled failure used for biological function [12].

\section{Eukaryotic flagellar dynamics and flow fields}

Eukaryotic flagella and cilia constitute one of the most highly conserved apparatuses in biology, their structure being virtually identical across a range of species, from the flagella of human sperm cells and motile algae to the cilia associated with reef-building corals. These organelles fulfil crucial roles in development [32], motility [33], sensing [34], and transport [3]. Although structurally very similar, the number, placement and role of flagella and cilia vary substantially across species. Spermatozoa (e.g., human, bull, sea urchin) actuate individual flagella to push themselves through the surrounding fluid. The biflagellate alga Chlamydomonas reinhardtii possesses two flagella which execute a breaststroke motion, pulling the cell through the fluid. Larger ensembles of flagella facilitate the motion of multicellular organisms such as Volvox carteri [35], while carpets of cilia act collectively to transport mucus and expel pathogens such as Pseudomonas aeruginosa from the human respiratory tract [36], establish the left-right asymmetry in developing embryos [37], and enhance mass transport in reef corals [7].

Flagella and their shorter versions - cilia - are filamentous structures composed of nine pairs of microtubule doublets bundled together, with two more microtubule singlets situated in the centre [38]. Collectively, these structures represent the axoneme. The peripheral microtubule doublets are linked together through various proteins including the two-headed molecular motor dynein. These motors quite literally "walk" along the microtubules [39], causing the constituent filaments to slide relative to one another and initiate bending waves in the flexible flagellum.

Although the structure associated with eukaryotic flagella is highly conserved, the waveforms exhibited by different species vary enormously. The flagella can be actuated to produce travelling waves, pushing fluid away from the cell, as in human or sea urchin sperm [40]. Other gaits display highly asymmetric beating profiles, which incorporate distinct power and recovery phases, producing the net motion of fluid $[41,42]$. In general, the temporal evolution of a flagellum's waveform is governed by a combination of active bending due to dynein motors (internal shear force), the elastic stiffness of the flagellum, and the externally imposed viscous load due to the relative fluid motion [43]. Simple models of coupled elastic filaments, sliding relative to one another as a result of prescribed oscillatory internal stresses, can qualitatively reproduce flagellar beating patterns [44]. The full oscillatory dynamics of flagellar waveforms, however, are likely to emerge from a more complex coupling between external load and the internal dynamics of the flagellum.

Characterising the spatiotemporal properties of the flows produced by individual beating flagella is important in quantifying their role in fundamental biological processes, and can help to decipher the emergent properties of ciliary arrays in a range of organisms. The disturbance caused by the motion of an arbitrary-shaped particle in low Reynolds number flow can be represented in terms of singularity solutions of the Stokes equations, Eq. (1). The velocity field $\mathbf{u}$ corresponding to a point force $\mathbf{F}$ at $\mathbf{x}=\mathbf{x}_{0}$ can be written in the form

$$
u_{i}(\mathbf{x})=\frac{1}{8 \pi \mu} G_{i j}\left(\mathbf{x}, \mathbf{x}_{0}\right) F_{j},
$$


where $G_{i j}$ is the Green's function [45]. The nature of the flow depends strongly on the topology of the fluid domain. For an unbounded, three-dimensional domain, the solution in Eq. (3) and the corresponding pressure field are given by [46] $u_{i}^{S}=\frac{F_{j}}{8 \pi \mu}\left(\delta_{i j} / r+r_{i} r_{j} / r^{3}\right)$ and $p^{S}=F_{j} r_{j} /\left(4 \pi r^{3}\right)$ respectively, where the vector $\mathbf{r}=\mathbf{x}-\mathbf{x}_{0}, r=|\mathbf{r}|$ and $\delta_{i j}$ is the usual Kronecker delta. This represents the leadingorder far-field disturbance created by a particle exerting a net force on the fluid, and is referred to as a Stokeslet.

The fluid disturbances created by flagella can be modelled mathematically through representation as a continuous distribution of point forces acting on the fluid [47]. Given a prescribed flagellar waveform, this approach [33] has been tremendously powerful for calculating the flows generated by beating flagella, and represents an effective technique for computing swimming speeds and power consumption $[48,49]$. Sophisticated tracking software can be used to identify the waveform frame by frame from extended movies of flagellar motion, enabling the automated calculation of the forces exerted on the fluid. Recent examples include isolated Volvox flagella [50], immobilised [51] and freely-swimming [52-54] Chlamydomonas cells and the flagella of bull sperm $[55]$.

In the far-field limit, the flow produced by a flagellum at each instant in time is equivalent to that of a single point force, and the dynamic waveform need not be considered. This simplification is of great value mathematically, permitting analytical solutions for the flow in systems of multiple flagella. Yet, it has remained unclear under what conditions the entire flow field associated with a beating flagellum can be represented by a single point force, and when higher order multipole contributions must be considered [56].

Quantification of the flow field associated with freely-swimming Chlamydomonas cells in a 3D chamber revealed that the time-averaged flow can be captured accurately using a three-Stokeslet model, in which the cell body and each of the two flagella are considered to exert a constant, stationary point force on the fluid [57] (Fig. 3A-B). The simple Stokeslet representation was able to accurately capture the salient topological features of the flow field. Guasto and coworkers were able to fully resolve the time-dependent flow fields associated with Chlamydomonas cells, swimming in a thin $2 \mathrm{D}$ film in order to prevent cell body rotation [58]. Significant topological changes in the fluid velocity occurred through the flagellar beating cycle (Fig. 3C-E). The time-resolved measurements of the dissipated power were more than 4 times higher than the predictions based on mean swimming speed, indicating the importance of capturing the unsteady components of flagella-driven flows.

Perhaps the most intuitive approach to characterising the intrinsic flow fields associated with eukaryotic flagella is to study the flow associated with just one isolated flagellum. Brumley and coworkers removed individual flagellated somatic cells from the colonial alga Volvox carteri, captured and oriented them using micropipettes, tracked the flagellar waveform and reconstructed the flow fields using particle image velocimetry [50]. The magnitude of the time-averaged flow field decayed as $\sim 1 / r$, consistent with the expected leading order Stokeslet contribution to the flow. However, this scaling was observed at distances as little as $r \gtrsim 20 \mu \mathrm{m}-$ comparable to the length of the flagellum itself. Moreover, the time-dependent flow field (Fig. 3F-G) was shown to be well represented by that of a time-varying point force (Fig. 3H-I). This demonstrates that the disturbance created by the nonreciprocal motion of a flagellum can be accurately represented as the motion of a sphere being pulled along a closed loop trajectory in a viscous fluid, an assumption often employed in models of flagellar motion (Sect. 4.2), and one which often enables the collective dynamics of multiple flagella to be studied mathematically in an analytical fashion $[13,59-61]$. 


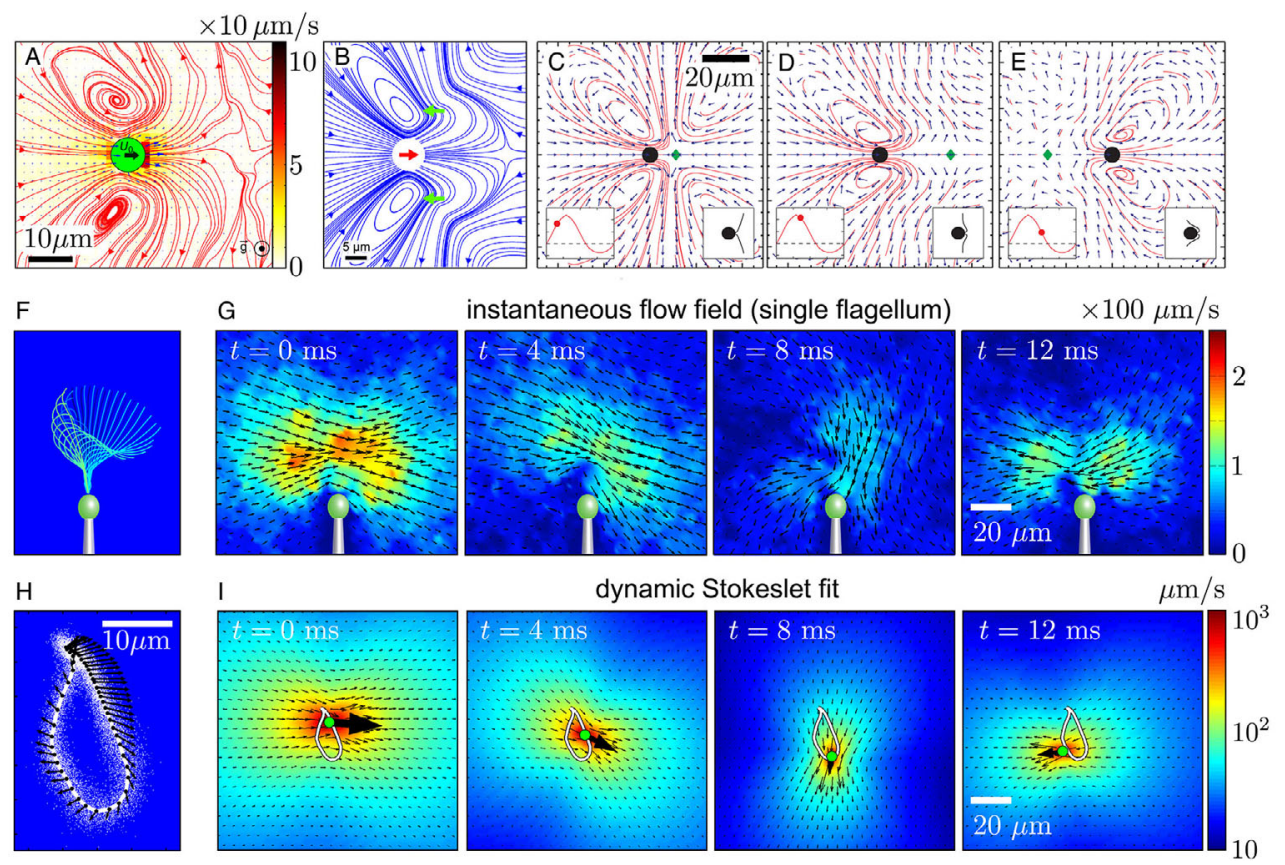

Fig. 3. Flagellar flow fields. (A) Time-averaged flow field of a freely-swimming Chlamydomonas cell, and (B) the three-Stokeslet model predictions. Reprinted figures with permission from Drescher, K., Goldstein, R. E., Michel, N., Polin, M., and Tuval, I. (2010) Physical Review Letters 105(16), 168101, dx.doi.org/10.1103/PhysRevLett.105.168101, by the American Physical Society [57]. (C-E) Snapshots (separated by approximately $6.3 \mathrm{~ms}$ ) of the oscillatory flow generated by Chlamydomonas as it swims in a thin 2D film. Reprinted figures with permission from Guasto, J.S., Johnson, K.A., and Gollub, J.P. (2010) Physical Review Letters 105(16), 168102, dx.doi.org/10.1103/PhysRevLett.105.168102 by the American Physical Society [58]. (F) Tracked waveform of an individual beating flagellum, isolated from Volvox carteri and (G) the time-dependent flow field it generates [50]. This flow is well characterised by a time-varying Stokeslet of variable direction and magnitude, traversing a closed-loop trajectory (H-I). Panels (F-I) are reproduced from Brumley et al. (2014) eLife 3, $\mathrm{e} 02750$.

Direct measurement of flagellar waveforms revealed that both the waveform shape evolution [50,62] and the beating phase [63] exhibit stochastic fluctuations. At the biomolecular level, this stochasticity can arise from random variations in the binding/unbinding of motor proteins in the axoneme, and is also influenced by calcium concentration [64]. The ability to resolve individual flagella for thousands of consecutive beats has enabled quantification of this stochasticity, the nature of which is important for synchronization properties of multiple flagella, as we shall see in Sect. 4.1.

Flagellar beating is also influenced by the surrounding physical microenvironment. The waveform of an individual isolated flagellum exhibited a characteristic shift when placed close to, but not touching, a dummy cell with no flagellum. Changes in the viscous drag arising from the presence of a nearby cell were enough to modify the intrinsic beating dynamics of the flagellum, indicating a subtle interplay between the internal dynamics, flagellar elasticity and external loading, in determining the overall waveform [50]. 


\section{Collective flagellar dynamics}

Whether in the human respiratory tract, on the surface of the protozan Opalina [65], or in green algae [13], arrays of eukaryotic cilia and flagella routinely exhibit striking coordinating in the form of metachronal waves - long-wavelength modulations of their beating phase. Metachronal waves occur when neighbouring cilia or flagella beat with a non-zero phase difference. For a two-dimensional ciliated surface, the propagation of this wave may be in any direction relative to the direction of the effective stroke. Metachronal waves are symplectic in nature when the direction of wave propagation is the same as that of the effective stroke of the flagella, and antiplectic metachronal waves occur when these directions are opposite [66].

Attempts to understand the mechanisms leading to metachronal coordination have been hampered by the lack of organisms suitable for the systematic study of flagella and the flows they create. Ensembles of cilia frequently occur with a high density, and are thus difficult to observe and manipulate. Identification of the fundamental physical mechanisms responsible for the spatiotemporal coordination of cilia is made more challenging by the high ciliary density, which means they are operating in the strong-coupling limit.

\subsection{Pairs of flagella}

Given the accuracy with which intrinsic flagellar flow fields can be represented by a single Stokeslet traversing a closed loop trajectory [50] (Fig. 3G-I), the class of models involving spherical colloidal oscillators represents a fruitful path for studying the collective motions of cilia and flagella. By modelling the two flagella of Chlamydomonas as spherical beads, Friedrich and Jülicher showed that they may achieve perfect synchrony through the rocking motion of the freely-swimming cell and local hydrodynamic friction forces [67]. These results were further supported with more detailed simulations [68], and in this fashion, the authors showed that synchronization can occur without dominant hydrodynamic interactions. However, the fact that flagella of Chlamydomonas and Volvox tend to synchronize even when the organisms are held perfectly still by a micropipette, demonstrates that hydrodynamic coupling does indeed play a significant role [69-71].

We consider now the synchronization properties of two colloidal oscillators situated in a quiescent fluid. If these spheres execute fixed trajectories in an unbounded fluid, each sphere possesses one degree of freedom, namely its phase $\phi_{i}$. Synchronization of two spheres will occur only if the phase difference $\Delta \phi=\phi_{2}-\phi_{1} \rightarrow 0$ as $t \rightarrow \infty$. The reversibility of Stokes flow implies that this synchronized state would be unstable if time were reversed. However, if the dynamical equation is invariant under exchange of $\phi_{1}$ and $\phi_{2}$ - as is the case for interacting Stokeslets - then the phase difference cannot undergo a net change over time [72]. Additional degrees of freedom must be introduced to break the time reversal symmetry and permit synchronization.

A number of mechanisms have been identified which facilitate synchronization of pairs of colloidal oscillators in different geometries. Spheres actuated by a constant driving force along fixed trajectories above a no-slip wall can exhibit phase-locking provided all symmetries in their spatial configuration are broken [73]. However, this mechanism is extremely sensitive to small perturbations in the intrinsic flagellar properties and configuration, and cannot account for the rapid and robust synchronization observed in biological systems [74], especially in the presence of noise and flagellar heterogeneities. For spheres driven along fixed trajectories in an unbounded fluid, synchronization will only occur when the spheres are driven internally by a nonconstant driving force $[60,75]$. Similar conclusions have been found for pairs of rigid rotating 
helices [76]. However, the constraint on the driving force can be relaxed if the oscillators are able to modify their beating pattern through hydrodynamic interactions [77]. Niedermayer and coworkers represented nodal cilia as widely separated spheres moving along circular trajectories of variable radius [59]. Since the spheres were subjected to a constant driving force, the angular velocity was radius-dependent. This extra degree of freedom provided a rapid and robust method of achieving synchrony. Simulations of rotating helices also reveal the importance of flexibility in achieving synchronization [78].

Modulations in the intrinsic driving force and the flexibility of trajectories are together responsible for the strong synchronization of flagella represented as colloidal oscillators, with the relative contributions from the two mechanisms depending on the precise configuration of the system [79]. Despite this, it remains unclear which of these contributions is most influential for systems of real flagella.

The most robust method for elucidating the mechanisms behind flagellar coordination involves the in situ study of pairs of flagella. With the biflagellate alga Chlamydomonas immobilised on a micropipette, Polin and coworkers studied the dynamics of their flagella for extended periods of time, discovering periods of synchronous beating interrupted with brief phase slips $[69,70]$. While the results are consistent with hydrodynamically mediated synchronization [71], the fact that both flagella emerge from the same cell means that other mechanisms could also be at play. Indeed, the recent observation of antiphase synchronization in $p t x 1$ - a non-phototactic mutant of Chlamydomonas [80] - points to the additional role of intracellular coupling.

To disentangle the hydrodynamic from the intracellular contributions to flagellar coordination, Brumley and coworkers performed experiments in which two flagellated somatic cells, isolated from the alga Volvox carteri, were each captured using micropipettes, oriented so that their beating planes coincided, and held at a controllable distance [50] (Fig. 4A-B). In this fashion, the two flagella, which exhibited distinct beating frequencies in isolation, were coupled directly and solely through the surrounding fluid. The configuration of this experiment can be viewed as a natural generalisation of previous work involving vibrating micropipettes [81] or microneedles [82]. With the knowledge that the strength of the flow produced by each flagellum exhibits a spatial decay according to $|\mathbf{u}| \sim 1 / r$, it was possible to test the hypothesis that cells sufficiently close to one another could synchronize their motion purely through mutual interaction of the flow fields they generate.

High-speed movies were recorded at various cell-cell spacings, $L=d / l$, and the flagellar phases $\phi_{1,2}$ were extracted using Poincaré sectioning of the dynamics $[69,70]$. The time-dependent interflagellar phase difference $\Delta(t)=\left(\phi_{1}-\phi_{2}\right) / 2 \pi$ was then used to characterise the synchronization properties. For widely separated cells (typically $L \gtrsim 4)$, the hydrodynamic interactions between the flagella are extremely weak, and each beats at its intrinsic frequency. Under these conditions, the phase difference drifts almost linearly with time, according to frequency mismatch (black curve of Fig. $4 \mathrm{C}$ ). For intermediate values of $L$, the flagella exhibit short periods of synchrony, interrupted by phase slips (blue and green curves of Fig. 4C). However, when the two cells are held close to one another $(L \lesssim 1)$, but without touching, the flagella exhibit uninterrupted synchrony for thousands of consecutive beats (red curve of Fig. 4C). This demonstrates that hydrodynamic interactions alone can facilitate robust phaselocking of flagella, even for pairs with markedly different natural frequencies (as much as $10 \%$ difference).

The phase dynamics are well-captured using the stochastic Adler equation $\dot{\Delta}=\delta \nu-U^{\prime}(\Delta)+\xi(t)$, where $\delta \nu$ is the intrinsic frequency difference between the two oscillators, $U$ is an effective potential which has period one in $\Delta$, and $\xi(t)$ is a Gaussian noise term with $\langle\xi(t)\rangle=0$ and $\left\langle\xi(t) \xi\left(t^{\prime}\right)\right\rangle=2 T_{\text {eff }} \delta\left(t-t^{\prime}\right)$ [69,70]. To leading order, 

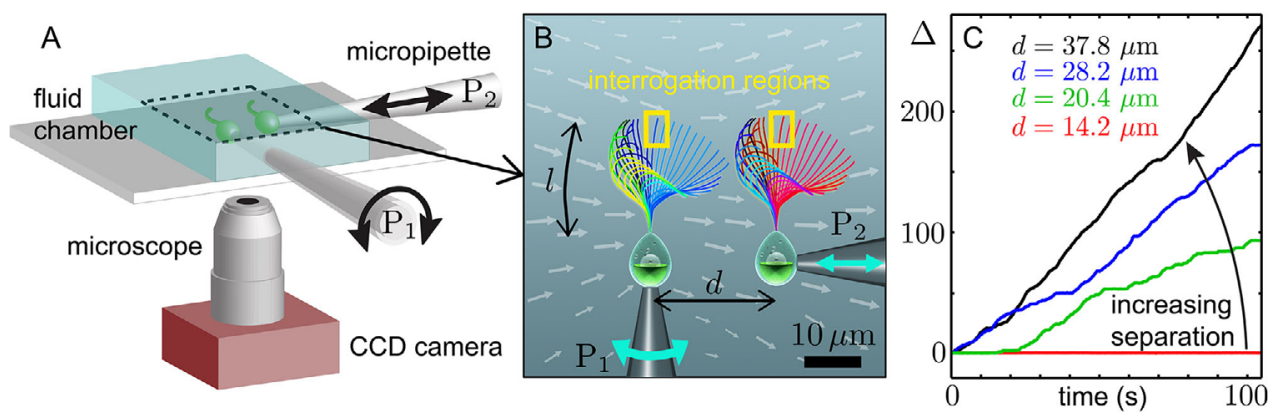

Fig. 4. Synchronized pairs of beating flagella. (A) Experimental apparatus in which two flagellated somatic cells from Volvox carteri are captured using micropipettes. (B) The separation $d$ and relative orientation of the two cells can be controlled using pipettes $P_{1}$ and $P_{2}$, which are attached to motorised micromanipulators. (C) Time-series showing interflagellar phase difference $\Delta=\left(\phi_{1}-\phi_{2}\right) / 2 \pi$ for one pair of cells at different separations [50]. Panels (A) and (C) are reproduced from Brumley et al. (2014) eLife 3, e02750.

$U=-\epsilon \cos (2 \pi \Delta)$, where $\epsilon$ is the interflagellar coupling strength, which can be extracted for each video recorded. The non-dimensionalised coupling strength, $\kappa=\epsilon / \bar{\omega}$, is captured accurately by the scaling $|\kappa| \propto L^{-1}$, in agreement with the quantified strength of the intrinsic time-dependent flagellar flow fields [50]. Taken together, these results demonstrate the importance of microscale fluid flows in facilitating the synchronous beating of eukaryotic flagella.

In the context of colloidal oscillator models, robust synchrony can be achieved through a phase-dependent driving force or an elastic trajectory [79]. Models of coupled flagella involving semiflexible filaments [83-85] also show a general tendency towards synchrony, but the precise role of flexibility remains unclear. Through dynamic tracking, Brumley and coworkers followed the flagellar waveform for pairs of cells and quantified the changes in the beating profile. For the same pair of cells at different separations, the flagella exhibited measurably different waveforms, revealing the importance of elasticity, and the need to study the bending properties, intrinsic active forces and external loading in a unified fashion [50]. For two colloidal oscillators in an unbounded fluid, separated by a distance $L$, the coupling strength can be written as

$$
\kappa_{\text {spheres }}=\frac{27 \mu \pi a^{2} l^{2} \bar{\omega}}{2 R} \frac{1}{L},
$$

where $a$ is the sphere radius, $R$ is the flagellar bending rigidity, $l$ the flagellar length, and $\bar{\omega}$ is the average beat frequency [50]. Based on realistic parameters for eukaryotic flagella, these elastic oscillators exhibit a coupling strength of $\kappa_{\text {spheres }}=0.014 \times L^{-1}$, close to the results for real Volvox flagella $\left(\kappa=0.016 \times L^{-1}\right)$ [50]. Including a phasedependent driving force in the context of this model did not affect the dynamics, with elasticity being overwhelmingly responsible for the drive towards synchrony in this parameter regime.

\subsection{Arrays of flagella}

Many theoretical studies [86-95] hint at a higher efficiency of propulsion by metachronal waves, and suggest a possible selective advantage in strategies to stabilise their occurrence. Motivated by experimental observations that the flagella of bull spermatozoa tend to synchronize when swimming close to one another [96], Taylor showed that for swimming sheets, energy dissipation is minimised in the in-phase 
state [97]. Similar conclusions have been obtained in studies of flexible sheets [98] and two-dimensional swimming cells [99]. While the presence of metachronal waves may in some way minimise the expenditure of energy, this principle provides limited insight into the physical mechanisms behind their emergence and the selection of wave patterns.

Early reports of correlated changes in the directions of flagellar beating and metachronal wave propagation $[100,101]$ led to the hypothesis of a mechanical origin for synchronization, and motivated theoretical studies involving hydrodynamically coupled filaments driven by various types of internal engines. Machin considered the nonlinear contractile elements of flagella [102], showing that synchronization was possible. Guirao and Joanny considered the semiflexible nature of flagella and modelled the internal motors governing the relative motion of microtubule doublets [84]. Yang and coworkers represented the filamentous structure of sperm flagella by long chains of spherical particles to investigate the synchronization properties [85]. While all of these models show a general tendency towards metachronal coordination, and help to explain the generation and regulation of flagellar motion subject to specific forces [103-106], their complexity prevents a simple understanding of the mechanisms responsible for collective motions.

Minimal models of hydrodynamically coupled self-sustained oscillators with few degrees of freedom give insight into the emergence of metachronal waves $[59,61,77$, $107,108]$, since in many cases the equations of motion can be solved explicitly. Such synchronization phenomena have been investigated experimentally with rotating paddles in viscous fluids [109], light driven microrotors [110] and colloids in optical tweezers $[79,111]$. These experiments confirm that hydrodynamic synchronization can be achieved even for relatively simple configurations.

Owing to its large size and ease of visualisation, the colonial alga Volvox carteri represents an ideal model system for studying collective flagellar dynamics and metachronal waves $[13,61]$. Indeed, many species of green algae have proven useful in fundamental studies of biological fluid mechanics [112]. Volvox is composed of thousands of biflagellate cells, embedded in a spherical extracellular matrix of radius $\sim 200 \mu \mathrm{m}$, and each beating their flagella towards the colony posterior (Fig. 5A). The surface-mounted somatic cells are a few tens of microns apart, and their flagella are therefore more nearly in the weak coupling limit than the cilia of Paramecium. At this separation, it is appropriate to model the fluid disturbance as a multipole expansion, keeping only the Stokeslet component [50] (Sect. 3).

Using particle image velocimetry, Brumley and coworkers measured the timedependent fluid velocity around Volvox colonies (Fig. 5B), and these results were used to characterise in detail the flagellar coordination [13,61]. These systems exclusively exhibited symplectic metachronal waves - in which the wave direction coincided with the direction of the flagellar power stroke (see kymograph in Fig. 5C). The metachronal waves were found to be noisy, and despite the robustness of their average properties, were punctuated by periodic phase defects (identified by white circles in Fig. 5C), during which synchrony was partial and limited to specific groups of cells [13].

To reconcile the emergent dynamics of these ensembles [61] with the known properties of individual flagella [50], an elastohydrodynamic model for interacting flagella was used, in which the action of each flagellum was represented by a sphere, situated above a no-slip wall, elastically bound to a circular trajectory, and driven around by a tangential driving force $f^{\text {drive }}$. For each rotor, the velocity $\mathbf{v}$ satisfies the force balance requirement of Stokes flow:

$$
\gamma(\mathbf{x}) \cdot \mathbf{v}=-\lambda\left(r-r_{0}\right) \mathbf{e}_{r}-\eta \zeta \mathbf{e}_{\zeta}+f^{\text {drive }} \mathbf{e}_{\phi} .
$$

Here, $\gamma=\gamma(\mathbf{x})=\gamma_{0}\left[\mathbf{I}+(9 a / 16 z)\left(\mathbf{I}+\mathbf{e}_{z} \mathbf{e}_{z}\right)+\mathcal{O}\left((a / z)^{3}\right)\right]$ is the friction tensor associated with the motion of the sphere near the no-slip wall, and $\gamma_{0}=6 \pi \mu a$ is the drag on a 


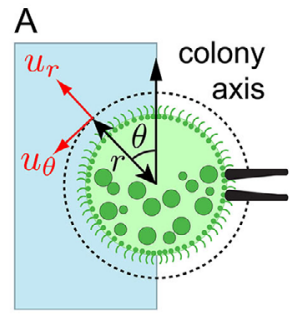

C
B

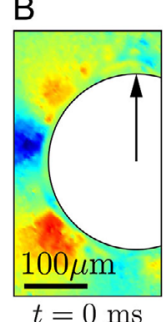

radial fluid velocity $\bar{u}_{r}$

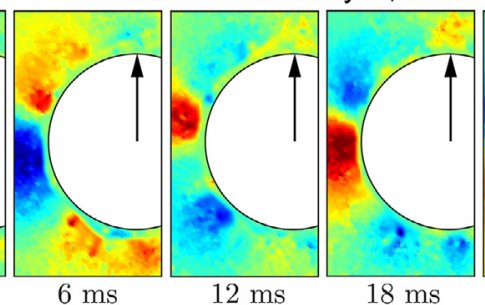
$\mu \mathrm{m} / \mathrm{s}$

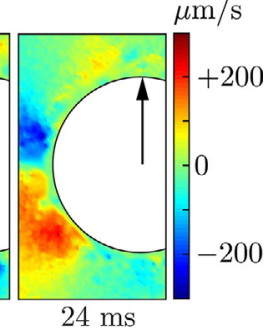

$\times 100 \mu \mathrm{m} / \mathrm{s}$
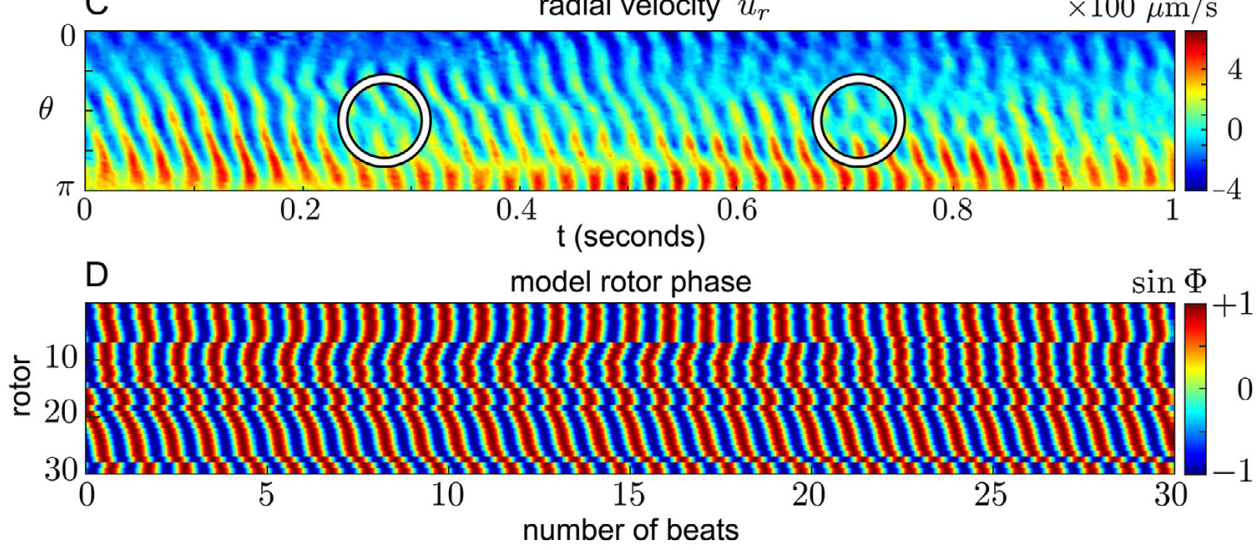

Fig. 5. Metachronal waves in arrays of flagella. (A) A Volvox colony held by a micropipette showing flagellated somatic cells (small dots) and interior daughter colonies (large circles). The dashed line indicates the distance at which the kymographs for the radial fluid velocity, $u_{r}$, is measured. (B) Snapshots of the time-dependent flow field, measured at various points throughout one flagellar beat. (C) Radial velocity $u_{r}\left(r^{*}, \theta, t\right)$, measured at $r^{*}=1.3 \times R$, where $R$ is the colony radius. Symplectic metachronal waves are clearly visible, interrupted periodically with phase defects (white circles). (D) Emergent properties of 30 hydrodynamically coupled model rotors, arranged in a linear configuration above a no-slip boundary. The model predicts symplectic metachronal waves, with a wavelength comparable to those observed experimentally, provided the orbits of the rotors are sufficiently flexible $(\Lambda=0.1$ in this case). This figure is reproduced with the permission of D.R. Brumley, M. Polin, T.J. Pedley, R.E. Goldstein, J. R. Soc. Interface 12, 20141358 (2015) [13].

sphere of radius $a$ in an unbounded fluid of viscosity $\mu$. For a system of $N$ rotors, the net hydrodynamic force acting on the $i^{\text {th }}$ rotor is given by

$$
\mathbf{F}_{i}=-\gamma_{i} \cdot\left[\mathbf{v}_{i}+\sum_{j \neq i} \mathbf{G}\left(\mathbf{x}_{j}, \mathbf{x}_{i}\right) \cdot \mathbf{F}_{j}\right]
$$

where $\mathbf{G}\left(\mathbf{x}_{j}, \mathbf{x}_{i}\right)$ is the Green's function coupling the different rotors in the presence of the no-slip wall (colony surface) [113]. The parameter of principal interest is the dimensionless ratio $\Lambda=\lambda d / f^{\text {drive }}$. This governs the deformability of the rotors' orbit: larger (smaller) values of $\Lambda$ mean stiffer (softer) radial recoil compared to the driving force, and result in smaller (larger) deviations from $r=r_{0}$. For Volvox flagella, the normalised spring constant is estimated as $\Lambda \sim 0.1[13,61]$. For pairs of rotors, the flow components perpendicular to the wall modify the in-phase configuration that would be stable in an unbounded fluid by compressing slightly the orbit of the upstream sphere, which speeds up, and dilating the orbit of the downstream one, which slows down. The stable equilibrium is then skewed to a negative phase lag. For linear arrays 
of 30 model rotors, designed to mimic the behaviour of cells along a single meridian of Volvox, the system naturally develops a symplectic metachronal wave with wavenumber $k \sim 2$ (Fig. 5D), in semiquantitative agreement with the experimental findings $(k=4.7 \pm 0.9)$. The recurring phase defects recently discovered in the experimental system [13] are also captured by this hydrodynamic model, and occur when there is a sufficiently steep local bias in the oscillator's intrinsic frequencies. Taken all together, these results demonstrate the importance of both the intrinsic flagellar compliance and interflagellar hydrodynamic interactions, in determining their global synchronization properties, and therefore the precise ways in which they interact with their environment.

\section{Dynamics of active swimmers in flow}

Understanding the physical interaction between microorganisms and ambient fluid flow [114] is beneficial for many applications, ranging from developing more accurate ecological models [115] to controlling and preventing the formation of biofilms [116]. However, the effect of the fluid dynamical environment on bacterial motility is still largely unexplored despite the ubiquity of flow in natural and artificial microbial habitats. Ambient flow impacts the ecology of microorganisms through a range of processes, including nutrient uptake, encounter rates, fertilisation and trophic interactions $[117,118]$. Additionally, soil microorganisms are frequently exposed to laminar groundwater flows [119] and the human microbiota endures flow in the gut and urinary system [120]. Investigating the effects of flow and shear stress on microorganisms is also of importance in many industrial processes, ranging from water desalination and wastewater treatment to the production of biofuels [121]. Shear, in particular, can influence chemotaxis and other biochemical processes crucial to the ecology of the microbial food web $[122,123]$, or play a fundamental role in the attachment of bacterial cells to surfaces, a critical step towards the formation of micro-colonies [124,125]. Moreover, unveiling the behaviour of a flowing suspension of self-propelled particles, besides being an interesting model of an out-of-equilibrium statistical system [126], could benefit the design and development of artificial micro-swimmers capable of serving as drug-delivery vehicles [127].

\subsection{Microorganisms in flow}

The motion of a single bacterium in a simple shear flow is the result of the intrinsic swimming motion of the cell, combined with the torque exerted on the cell by the local vorticity [8]. The coupling between locomotion and flow can lead to spatial heterogeneity in the population distribution of motile organisms. Zooplankton, for instance, often accumulate in the presence of strong vertical currents at frontal zones in the ocean [128]. Thin layers of motile phytoplankton cells can be formed by a balance between a preferred swimming direction and velocity gradients [129,130], which can ultimately cause convective instability phenomena for strong cell accumulation [131]. Sperm cells are known to preferentially swim upstream [132,133], likely as a result of the combined action of shear and hydrodynamic attraction towards a nearby wall [134]. Similarly, a weak Poiseuille flow can cause alignment of bacteria swimming above an open surface to create an upstream net flux [126,135], while at higher shear rates the balance between a lift force acting on the chiral shape of the flagellum and the increased drag around the cell body can generate a torque that reorients the swimming bacterium in a direction perpendicular to the plane of 
shear [136]. These examples illustrate the influence of fluid flow on microbial motility, and highlight the importance of studying the transport of microorganisms within confined geometries designed to mimic catheters, blood vessels, porous media and environmental microscale flows.

In the absence of inertial effects, the centroid of a passive rigid particle follows the streamlines of the underlying flow while the body undergoes periodic orbits known as Jeffery orbits [137]. In a Jeffery orbit, an elongated particle is aligned for most of the time with the flow direction and rotates at a non-constant rate with a frequency that is proportional to the local shear rate and inversely proportional to the particle's aspect ratio. In contrast, "active" particles - where the activity can be of chemical, mechanical or most commonly biological origin, as in the case of bacteria can change their position relative to a flowing fluid and are not bound to follow streamlines. These dynamics have recently attracted considerable attention from physicists, mostly in the theoretical domain, because the dynamics of active particles in flow can produce rich and unexpected behaviours. Examples of such effects include the clustering of elongated self-propelled particles around chaotic trajectories in cellular laminar flows [138]. Counterintuitively, the motility of active particles does not necessarily lead to enhanced transport; rather, for small but finite values of the swimming speed, motile particles can be trapped for a very long time near the boundaries between chaotic and regular flow regions [139]. Although the accumulation of swimmers in simple cellular or vortical flows is significantly reduced in a realistic threedimensional environment, elongated self-propelled particles show a level of clustering that exceeds that of a Poisson distribution in turbulent flow [140].

Studies investigating the dynamic behaviour of single self-propelled particles in an imposed shear flow are of increasing interest to both theoretical and experimental communities. Mean trajectories and the mean square displacement of particles can show different long-time behaviours depending on the presence of shear and if Brownian motion is taken into account [141]. In the absence of noise, microswimmers are predicted to follow specific trajectories in a Poiseuille flow [142,143]. Depending on the aspect ratio and the flow strength, a rigid self-propelled particle is predicted to either swim swinging around the centreline of the channel or to constantly tumble in the regions of higher shear rate towards the sidewalls of the channel (Fig. 6A). This scenario becomes more complex if one takes into account the flexibility of bacterial flagella [144], in which case swimmers are predicted to migrate towards the centreline of the channel and swim against the flow in a limit-cycle trajectory (Fig. 6B). Numerical simulations have shown that the amplitude of the oscillations around the centreline depends on the flexibility of the flagellum. Testing these theoretical predictions has remained elusive owing to the challenge of resolving trajectories of individual bacteria in precisely controlled fluid flows. Recently, this challenge has been overcome with the use of microfluidic devices, which simultaneously allow high-resolution observations of individual bacterial trajectories and the spatial distribution of bacterial populations in carefully controlled, laminar fluid flows (Fig. 6C) [1,8]. Below we describe recent experimental observations in this domain.

The dynamics of eukaryotic cells - such as phytoplankton - in flow are also affected by multiple features pertaining to cell shape and motility. For example, flagellar beating can modify the effective hydrodynamic aspect ratio of the cell compared to the cell body alone [145], impacting the cell's rotational dynamics. Furthermore, the question of whether eukaryotic cells can actively modify their swimming behaviour in response to fluid shear remains wide open, and would add entirely new dynamics to the system. In particular, certain phytoplankton species exhibit a directional persistence in shear flows [146] that is inconsistent with predictions for passive particles undergoing Jeffery orbits and suggests that cells can actively resist the viscous torques induced by the shear flow [147]. While conclusive evidence that the response is active 

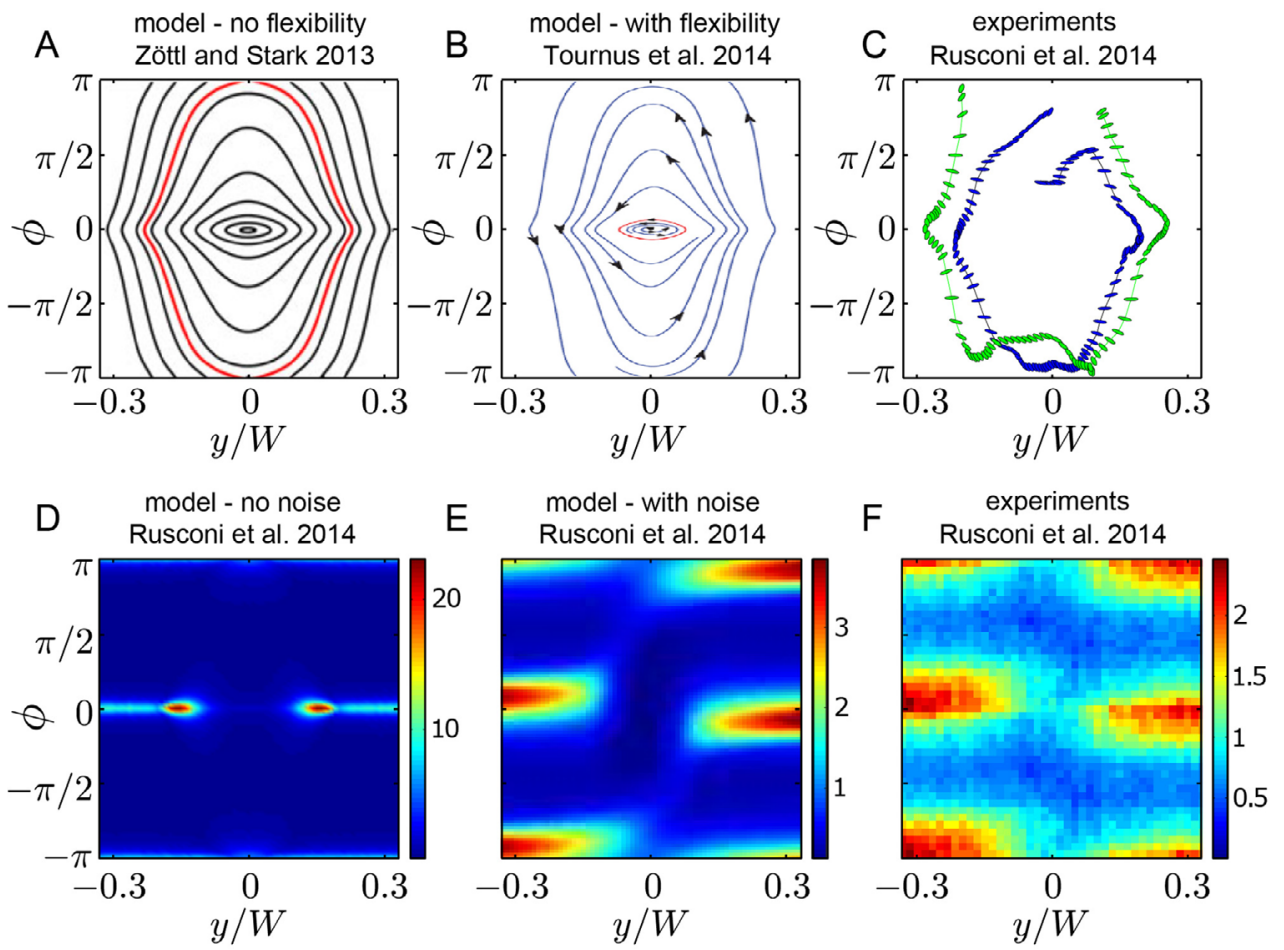

Fig. 6. Theoretical and experimental dynamics of self-propelled particles and bacteria in a planar Poiseuille flow are represented by (A-C) phase diagrams of swimming trajectories and (D-F) bacterial concentration. (A) Particle orientation, $\phi$, and normalised cross-stream position $(y / W)$ for an idealised elongated microswimmer; the red line is the separatrix dividing swinging and tumbling motion. A. Zöttl, H. Stark, Eur. Phys. J. E 36, 1 (2013) [143]. (B) Same as A but assuming a flexible flagellum; the red line corresponds to a limit cycle towards which the swimmer slowly converges. Tournus, M., Kirshtein, A., Berlyand, L.V., and Aranson, I.S. (2015) Journal of The Royal Society Interface 12(102), 20140904, by permission of the Royal Society [144]. (C) Trajectories of smooth-swimming Bacillus subtilis in a microfluidic Pouiseuille flow. (D) Langevin model results for the normalised cell concentration in the absence of rotational noise $\left(D_{R}=0\right)$ [14]. (E) Langevin model results for noisy swimmers $\left(D_{R}=1 \mathrm{rad}^{2} \mathrm{~s}^{-1}\right)$. (F) Experimental quantification of the normalised cell concentration for wild-type B. subtilis under similar conditions to E. Panels (C-F) reprinted by permission from Macmillan Publishers Ltd: Rusconi, R., Guasto, J.S., and Stocker, R. (2014) Nature Physics 10(3), 212217, doi: 10.1038/NPHYS2883.

is lacking and difficult to obtain, these observations are intriguing in that they show that the coupling between motility and flow may have a biological dimension that makes the dynamics even richer than the purely passive coupling of swimmers and flow.

\subsection{Shear trapping of motile cells}

The effect of shear on swimming bacteria can result in strong heterogeneities in their spatial distribution (Fig. 6D-F). In recent experiments, Rusconi and coworkers subjected different bacterial species (Bacillus subtilis and Pseudomonas aeruginosa) to a large range of flow rates in a $50 \mathrm{~cm}$-long microfluidic device. The height of the channel $(H=750 \mu \mathrm{m})$ was approximately twice its width $(W=425 \mu \mathrm{m})$, and so the velocity 
profile was parabolic along the width of the channel. Imaging occurred at mid-depth using high-speed video microscopy, simultaenously recording the trajectories of vast numbers of bacteria. Without flow, the density distribution of a dilute suspension of smooth-swimming B. subtilis (i.e., a mutant that is incapabale of tumbling) was uniform. In contrast, in the presence of flow the cell concentration showed the formation of a strong depletion zone near the centre of the channel and accumulation towards the lateral walls, and this effect increased with increasing flow rate or, equivalently, shear rate. This "shear trapping" phenomenon originated from cell motility, as dead cells did not show any deviation from a uniform distribution at any shear rate.

The spatial non-uniformity in the distribution of bacteria grew with increasing shear rate, up to a maximum at shear rates of $\sim 10 \mathrm{~s}^{-1}$, before decreasing at larger shear rates [14]. The same shear trapping effect was observed for the wildtype strain of $B$. subtilis and for wild-type $P$. aeruginosa [14]. This indicates that the phenomenon is largely independent of the details of the propulsion system and the reorientation mechanism, because $B$. subtilis has multiple flagella and swims in a run-and-tumble pattern, whereas $P$. aeruginosa has a single polar flagellum and swims in a run-and-reverse fashion.

To better understand the origin of shear trapping, the two-dimensional dynamics of a single bacterium were studied in a parabolic flow through a mathematical model [14]. The bacterium was modelled as a rigid ellipsoid of aspect ratio $q$ and constant swimming speed $V$, oriented at an angle $\phi$ with respect to the direction of the flow. The cell's equations of motion in the same parabolic flow as in the experiments are then

$$
\begin{aligned}
& \dot{x}=-V \cos \phi+u(y) \\
& \dot{y}=-V \sin \phi \\
& \dot{\phi}=\frac{4 y U}{W^{2}}\left[1+\left(\frac{1-q^{2}}{1+q^{2}}\right) \cos 2 \phi\right]+\xi_{R},
\end{aligned}
$$

where $\xi_{R}$ represents rotational noise as a Gaussian-distributed angular velocity with zero mean and a variance proportional to the cell's effective rotational diffusivity $D_{R}$. The cell concentration profiles, obtained by solving numerically the equations of motion for a large number of swimmers, are in very good agreement with the experimental profiles, including the maximal depletion at mean shear rates of $2.5-10 \mathrm{~s}^{-1}$ (Fig. 6E-F). The model also reveals the role of cell body elongation, a characteristic common to essentially all motile bacteria because of the high aspect ratio of their flagella. Elongated swimmers exhibit preferential swimming alignment that results in trapping, whereas simulations with spherical swimmers exhibit no depletion. The maximum in bacterial depletion at an intermediate mean shear rate $S$, observed experimentally and confirmed by the numerical model, can be interpreted by considering the competition between the shear-induced alignment of elongated bacteria with the flow and the stochasticity in their swimming orientation, described by the rotational Péclet number, $\mathrm{Pe}=S / D_{R}$. Such analysis, corroborated by a Fokker-Plank model, has shown that the trapping effect grows as $\sim \mathrm{Pe}^{2}$ at low Péclet numbers and decreases as $\sim \mathrm{Pe}^{-0.5}$ at high Péclet numbers.

Rusconi and coworkers further demonstrated that shear trapping directly impacts bacterial survival strategies, as it can entirely suppress chemotaxis and enhance surface attachment $[8,14]$. Indeed, one important consequence of shear trapping on bacterial transport is its effect on the cell's ability to respond to external stimuli [148]. The effect of a spatially varying shear on aerotaxis was investigated, by imposing a steady linear oxygen gradient across the channel. In the absence of flow, the concentration of bacteria was skewed towards the side with the higher concentration of oxygen. Upon starting the flow through the channel, the bacterial density profiles pro- 
gressively shifted from their quiescent distribution and the shear-induced depletion and accumulation mechanism prevailed over the aerotactic response at increasing flow rates [14]. These experiments demonstrated the quenching effect of shear trapping on chemotaxis. In contrast, shear trapping was found to increase surface attachment [14]. In particular, when the mean shear was larger than $20 \mathrm{~s}^{-1}$ the surface coverage after $1 \mathrm{~h}$ of continuous flow was more than double the surface coverage in the absence of flow. With care taken to avoid artifacts that easily occur in surface attachment problems, the authors concluded that the origin of this enhanced surface attachment under flow was shear trapping, which creates an accumulation of cells in the near-surface region, and thus a higher flux of bacteria to the surface.

Similar experiments were recently performed with phytoplankton cells, by comparing the spatial distribution of four species of phytoplankton in flow [149]. At moderate shear rates $\left(>1 \mathrm{~s}^{-1}\right)$, heterogeneous distributions of cells were again observed. These experiments revealed that the spatial distribution of phytoplankton in flow depends not only on the fluid shear rate and the cell's aspect ratio, as in the case of bacteria, but also on the cell's swimming mode, in particular whether cells were pushers or pullers. By computing the effective aspect ratio - which included the contribution from the cell body shape, the protruding flagella, and the flagellar beat pattern - it was found that cells with higher effective aspect ratios tended to accumulate in the higher shear regions of the flow, a result consistent with the shear trapping mechanism observed in bacteria. However, for shear rates greater than $10 \mathrm{~s}^{-1}$, biflagellate breaststroke swimmers were found to accumulate in the regions of the microchannel characterised by relatively low shear, suggesting an active response to shear or a coupling between shear and flagellar beating. These observations encompass a rich set of dynamics that emerge from the interaction between phytoplankton motility, morphology and fluid flow, and open a new class of problems in biological fluid dynamics at the interface between microbial ecology and the physics of active suspensions.

\section{Outlook}

Eukaryotic and prokaryotic flagella, such as those found in the algae Volvox and the bacterium $V$. alginolyticus, respectively, exhibit gaits that are not fixed, but rather depend on physical and chemical conditions of the environment. As a consequence of the elasticity of these systems, the observed dynamics emerge from the interactions between external fluid flows and the flexible cell appendages. Experimentally studying the delicate interplay between forces and torques arising from microscale fluid flows and the mechanical responses of individual microbes and their flexible appendages demands the use of precisely controlled environments, recently afforded through microfluidic technologies.

The ability to analyse microbial systems across a range of length and time scales is important in determining their overall ecological attributes. Reconciling the intrinsic mechanical properties of the bacterial flagellar hook with the overall trajectories of bacteria strengthens the handshake between experiment and theory, and provides a concrete base upon which studies of chemotaxis and collective motions can be built. Studying the dynamics of eukaryotic flagella individually, in pairs, and in large arrays, is the most intuitive way of elucidating the mechanisms behind their striking coordination. In the same fashion, the ability to simultaneously image individual motile microorganisms in prescribed ambient flows, and their dynamics at a population level over longer timescales, enables a deeper understanding of the coupling between fluid flow and microbial motility, and investigation of the flow-sensing capabilities of microbes. 
Understanding the roles that fluid mechanics, motility and flexibility play in the physical ecology of microorganisms depends largely on the ability to apply physical principles to the microbial world and to bring new observational tools to the challenging task of resolving fast and spatially heterogeneous microscale dynamics. In this respect, the recent influx of physicists into the microbial world and the advent of advanced observation techniques including microfluidics, promises a rich and intriguing future for this field and ultimately, it is hoped, the identification of fundamental physical mechanisms that are broadly applicable throughout the world of microorganisms.

The authors gratefully acknowledge support through a Human Frontier Science Program (HFSP) Cross-Disciplinary Fellowship (to D.R.B.), a Samsung Scholarship (to K.S.), and a Marine Microbiology Initiative Investigator Award from the Gordon and Betty Moore Foundation (GBMF3783, to R.S.). The authors also thank G. Gorick for help with some of the figures.

\section{References}

1. K. Son, D.R. Brumley, R. Stocker, Nat. Rev. Microbiol. 13, 761 (2015)

2. J. Elgeti, R.G. Winkler, G. Gompper, Rep. Prog. Phys. 78, 56601 (2015)

3. M.B. Short, C.A. Solari, S. Ganguly, T.R. Powers, J.O. Kessler, R.E. Goldstein, Proc. Natl. Acad. Sci. USA 103, 8315 (2006)

4. T. Ishikawa, M.P. Simmonds, T.J. Pedley, J. Fluid Mech. 568, 119 (2006)

5. R. Rusconi, S. Lecuyer, N. Autrusson, L. Guglielmini, H.A. Stone, Biophys. J. 100, $1392(2011)$

6. J. Brueckner, M. McGrath, Curr. Opin. Genet. Dev. 13, 385 (2003)

7. O.H. Shapiro, V.I. Fernandez, M. Garren, J.S. Guasto, F.P. Debaillon-Vesque, E. Kramarsky-Winter, A. Vardi, R. Stocker, Proc. Natl. Acad. Sci. USA 111, 13391 (2014)

8. R. Rusconi, R. Stocker, Curr. Opin. Microbiol. 25, 1 (2015)

9. K. Drescher,Y. Shen, B.L. Bassler, H.A. Stone, Proc. Natl. Acad. Sci. USA 110, 4345 (2013)

10. J. Li, G. Lykotrafitis, M. Dao, S. Suresh, Proc. Natl. Acad. Sci. USA 104, 4937 (2007)

11. J. Gore, Z. Bryant, M. Nöllmann, M.U. Le, N.R. Cozzarelli, C. Bustamante, Nature 442, 836 (2006)

12. K. Son, J.S. Guasto, R. Stocker, Nat. Phys. 9, 494 (2013)

13. D.R. Brumley, M. Polin, T.J. Pedley, R.E. Goldstein, J. R. Soc. Interface 12, 20141358 (2015)

14. R. Rusconi, J.S. Guasto, R. Stocker, Nat. Phys. 10, 212 (2014)

15. E.M. Purcell, Am. J. Phys. 45(1), 3 (1977)

16. H.C. Berg, R.A. Anderson, Nature 245, 380 (1973)

17. H.C. Berg, Annu. Rev. Biochem. 72, 19 (2003)

18. H.C. Berg, D.A. Brown, Nature 239, 500 (1972)

19. H.C. Berg, E. coli in Motion 1st edition (Springer-Verlag, New York, 2004)

20. E. Lauga (2015) [arXiv preprint] [arXiv: 1509. 02184] Annu. Rev. Fluid Mech, to appear (2016)

21. L. Turner, W.S. Ryu, H.C. Berg, J. Bacteriol. 182, 2793 (2000)

22. N.C. Darnton, L. Turner, S. Rojevsky, H.C. Berg, J. Bacteriol. 189, 1756 (2007)

23. S.E. Spagnolie, E. Lauga, Phys. Rev. Lett. 106, 58103 (2011)

24. M.T. Brown, B.C. Steel, C. Silvestrin, D.A. Wilkinson, N.J. Delalez, C.N. Lumb, B. Obara, J.P. Armitage, R.M. Berry, J. Bacteriol. 194, 3495 (2012)

25. E. Leifson, B.J. Cosenza, R. Murchelano, R.C. Cleverdon, J. Bacteriol. 87, 652 (1964)

26. L. Xie, T. Altindal, S. Chattopadhyay, X.-L. Wu, Proc. Natl. Acad. Sci. USA 108, $2246(2011)$ 
27. R. Stocker, J.R. Seymour, Microbiol. Mol. Bio. Rev. 76, 792 (2012)

28. E. Lauga, T.R. Powers, Rep. Prog. Phys. 72, 096601 (2009)

29. S.P. Timoshenko, Theory of elastic stability, 2nd edn. (McGraw-Hill, 1961)

30. G. Li, C.S. Smith, Y.V. Brun, J.X. Tang, J. Bacteriol. 187, 257 (2005)

31. E. Alipour-Assiabi, G. Li, T.R. Powers, J.X. Tang, Biophys. J. 90, 2206 (2006)

32. S. Nonaka, H. Shiratori, Y. Saijoh, H. Hamada, Nature 418, 96 (2002)

33. J. Gray, G.J. Hancock, J. Exp. Biol. 32, 802 (1955)

34. R. Shlomovitz, Y. Roongthumskul, S. Ji, D. Bozovic, R. Bruinsma, Interface Focus 4, $20140022(2014)$

35. K. Drescher, K.C. Leptos, I. Tuval, T. Ishikawa, T.J. Pedley, R.E. Goldstein, Phys. Rev. Lett. 102, 168101 (2009)

36. B. Button, L. Cai, C. Ehre, M. Kesimer, D.B. Hill, J.K. Sheehan, R.C. Boucher, M. Rubinstein, Science 337, 937 (2012)

37. J.H.E. Cartwright, O. Piro, I. Tuval, Proc. Natl. Acad. Sci. USA 101, 7234 (2004)

38. D.W. Fawcett, K.R. Porter, J. Morph. 94, 221 (1954)

39. A. Gennerich, R.D. Vale, Curr. Opin. Cell Biol. 21, 59 (2009)

40. R. Rikmenspoel, J. Cell Biol. 76, 310 (1978)

41. M.A. Sleigh, J. Exp. Biol. 37, 1 (1960)

42. J. Gray, Proc. R. Soc. B, 93, 104 (1922)

43. C.J. Brokaw, Nature 209, 161 (1966)

44. S. Camalet, F. Jülicher, J. Prost, Phys. Rev. Lett. 82, 1590 (1999)

45. C. Pozrikidis, Boundary integral and singularity methods for linearized viscous flow, (Cambridge University Press, Cambridge, 1992)

46. J.R. Blake, A.T. Chwang, J. Eng. Math. 8, 23 (1973)

47. G.J. Hancock, Proc. R. Soc. A. 217, 96 (1953)

48. J. Lighthill, SIAM Rev. 18, 161 (1976)

49. R.E. Johnson, C.J. Brokaw, Biophys. J. 25, 113 (1979)

50. D.R. Brumley, K.Y. Wan, M. Polin, R.E. Goldstein, eLife 3, e02750 (2014)

51. K.Y. Wan, R.E. Goldstein, Phys. Rev. Lett. 113, 238103 (2014)

52. P.V. Bayly, B.L. Lewis, P.S. Kemp, R.B. Pless, S.K. Dutcher, Cytoskeleton 67, 56 (2010)

53. P.V. Bayly, B.L. Lewis, E.C. Ranz, R.J. Okamoto, R.B. Pless, S.K. Dutcher, Biophys. J. 100, 2716 (2011)

54. H. Kurtuldu, D. Tam, A.E. Hosoi, K.A. Johnson, J.P. Gollub, Phys. Rev. E 88, 13015 (2013)

55. B.M. Friedrich, I.H. Riedel-Kruse, J. Howard, F. Jülicher, J. Exp. Biol. 213, 1226 (2010)

56. A. Vilfan, Eur. Phys. J. E 35, 1 (2012)

57. K. Drescher, R.E. Goldstein, N. Michel, M. Polin, I. Tuval, Phys. Rev. Lett. 105, $168101(2010)$

58. J.S. Guasto, K.A. Johnson, J.P. Gollub, Phys. Rev. Lett. 105, 168102 (2010)

59. T. Niedermayer, B. Eckhardt, P. Lenz, Chaos 18, 37128 (2008)

60. N. Uchida, R. Golestanian, Phys. Rev. Lett. 106, 58104 (2011)

61. D.R. Brumley, M. Polin, T.J. Pedley, R.E. Goldstein, Phys. Rev. Lett. 109, 268102 (2012)

62. K.Y. Wan, K.C. Leptos, R.E. Goldstein, J. R. Soc. Interface 11, 20131160 (2014)

63. R. Ma, G.S. Klindt, I.H. Riedel-Kruse, F. Jülicher, B.M. Friedrich, Phys. Rev. Lett. 113, $48101(2014)$

64. M. Bessen, R.B. Fay, G.B. Witman, J. Cell Biol. 86, 446 (1980)

65. S.L. Tamm, G.A. Horridge, Proc. R. Soc. B, 175, 219 (1970)

66. E.W. Knight-Jones, Quart. J. Microscop. Sci. 95, 503 (1954)

67. B.M. Friedrich, F. Jülicher, Phys. Rev. Lett. 109, 138102 (2012)

68. V.F. Geyer, F. Jülicher, J. Howard, B.M. Friedrich, Proc. Natl. Acad. Sci. USA 110, 18058 (2013)

69. R.E. Goldstein, M. Polin, I. Tuval, Phys. Rev. Lett. 103, 168103 (2009) 
70. M. Polin, I. Tuval, K. Drescher, J.P. Gollub, R.E. Goldstein, Science 325, 487 (2009)

71. R.E. Goldstein, M. Polin, I. Tuval, Phys. Rev. Lett. 107, 148103 (2011)

72. R. Golestanian, J.M. Yeomans, N. Uchida, Soft Matter 7, 3074 (2011)

73. A. Vilfan, F. Jülicher, Phys. Rev. Lett. 96, 58102 (2006)

74. D.M. Woolley, R.F. Crockett, W.D.I. Groom, S.G. Revell, J. Exp. Biol. 212, 2215 (2009)

75. N. Uchida, R. Golestanian, Eur. Phys. J. E 35, 1 (2012)

76. M. Kim, T.R. Powers, Phys. Rev. E 69, 61910 (2004)

77. P. Lenz, A. Ryskin, Phys. Biol. 3, 285 (2006)

78. M. Reichert, H. Stark, Eur. Phys. J. E 17, 493 (2005)

79. J. Kotar, L. Debono, N. Bruot, S. Box, D. Phillips, S. Simpson, S. Hanna, P. Cicuta, Phys. Rev. Lett. 111, 228103 (2013)

80. K.C. Leptos, K.Y. Wan, M. Polin, I. Tuval, A.I. Pesci, R.E. Goldstein, Phys. Rev. Lett. 111, 158101 (2013)

81. D. Eshel, I.R. Gibbons, Cell Mot. Cytoskel. 14, 416 (1989)

82. M. Okuno, Y. Hiramoto, J. Exp. Biol. 65, 401 (1976)

83. S. Gueron, K. Levit-Gurevich, N. Liron, J.J. Blum, Proc. Natl. Acad. Sci. USA 94, $6001(1997)$

84. B. Guirao, J.-F. Joanny, Biophys. J. 92, 1900 (2007)

85. Y. Yang, J. Elgeti, G. Gompper, Phys. Rev. E 78, 61903 (2008)

86. J. Elgeti, G. Gompper, Proc. Natl. Acad. Sci. USA 110, 4470 (2013)

87. S. Gueron, K. Levit-Gurevich, Proc. Natl. Acad. Sci. USA 96, 12240 (1999)

88. S. Gueron, N. Liron, Biophys. J. 63, 1045 (1992)

89. S. Gueron, N. Liron, Biophys. J. 65, 499 (1993)

90. S. Michelin, E. Lauga, Phys. Fluids 22, 111901 (2010)

91. N. Osterman, Vilfan, Proc. Natl. Acad. Sci. USA 108, 15727 (2011)

92. C. Mettot, E. Lauga, Phys. Rev. E 84, 061905 (2011)

93. A. Dauptain, J. Favier, A. Bottaro, J. Fluids Struct. 24, 1156 (2008)

94. J.R. Blake, J. Fluid Mech. 55, 1 (1972)

95. X. Yang, R.H. Dillon, L.J. Fauci, Bull. Math. Biol. 70, 1192 (2008)

96. Rothschild, Nature 163, 358 (1949)

97. G.I. Taylor, Proc. R. Soc. Lond. A, Math. 209, 447 (1951)

98. G.J. Elfring, E. Lauga, J. Fluid Mech. 674, 163 (2011)

99. G.J. Elfring, E. Lauga, Phys. Fluids 23, 11902 (2011)

100. C. Brennen, H. Winet, Annu. Rev. Fluid Mech. 9, 339 (1977)

101. H. Machemer, J. Exp. Biol. 57, 239 (1972)

102. K.E. Machin, Proc. R. Soc. B 158, 88 (1963)

103. I.H. Riedel-Kruse, A. Hilfinger, J. Howard, F. Jülicher, HFSP J. 1, 192 (2007)

104. C.J. Brokaw, J. Exp. Biol. 53, 445 (1970)

105. C.J. Brokaw, Biophys. J. 12, 564 (1972)

106. R. Rikmenspoel, J. Exp. Biol. 108, 205 (1984)

107. M.C. Lagomarsino, P. Jona, B. Bassetti, Phys. Rev. E 68, 021908 (2003)

108. C. Wollin, H. Stark, Eur. Phys. J. E 34, 42 (2011)

109. B. Qian, H. Jiang, D.A. Gagnon, K.S. Breuer, T.R. Powers, Phys. Rev. E 80, 61919 (2009)

110. R. Di Leonardo, A. Búzás, L. Kelemen, G. Vizsnyiczai, L. Oroszi, P. Ormos, Phys. Rev. Lett. 109, 034104 (2012)

111. J. Kotar, M. Leoni, B. Bassetti, M.C. Lagomarsino, P. Cicuta, Proc. Natl. Acad. Sci. USA 107, 7669 (2010)

112. R.E. Goldstein, Annu. Rev. Fluid Mech. 47, 343 (2015)

113. J.R. Blake, Math. Proc. Camb. Phil. Soc. 70, 303 (1971)

114. J.S. Guasto, R. Rusconi, R. Stocker, Annu. Rev. Fluid Mech. 44, 373 (2012)

115. A. Persat, C.D. Nadell, M.K. Kim, F. Ingremeau, A. Siryaporn, K. Drescher, N.S. Wingreen, B.L. Bassler, Z. Gitai, H.A. Stone, Cell 161, 988 (2015)

116. L. Hall-Stoodley, J.W. Costerton, P. Stoodley, Nat. Rev. Microbiol. 2, 95 (2004) 
117. F. Peters, C. Marrasé, H. Havskum, F. Rassoulzadegan, J. Dolan, M. Alcaraz, J. Gasol, J. Plankton Res. 24, 321 (2002)

118. J.R. Taylor, R. Stocker, Science 338, 675 (2012)

119. D. John, J. Rose, Environ. Sci. Techonol. 39, 7345 (2005)

120. H.J. Kim, D. Huh, G. Hamilton, D.E. Ingber, Lab on a Chip 12, 2165 (2012)

121. M.A. Bees, O.A. Croze, Biofuels 5, 53 (2014)

122. R.H. Luchsinger, B. Bergersen, J.G. Mitchell, Biophys. J. 77, 2377 (1999)

123. J.T. Locsei, Pedley, Bull. Math. Biol. 71, 1089 (2009)

124. W.E. Thomas, V. Vogel, E. Sokurenko, Annu. Rev. Biophys. 37, 399 (2008)

125. S. Lecuyer, R. Rusconi, Y. Shen, A. Forsyth, H. Vlamakis, R. Kolter, H.A. Stone, Biophys. J. 100, 341 (2011)

126. R. Nash, R. Adhikari, J. Tailleur, M. Cates, Phys. Rev. Lett. 104, 258101 (2010)

127. B.J. Nelson, I.K. Kaliakatsos, J.J. Abbott, Annu. Rev. Biomedical Eng. 12, 55 (2010)

128. A. Genin, J.S. Jaffe, R. Reef, C. Richter, P.J.S. Franks, Science 308, 860 (2005)

129. J.O. Kessler, Nature 313, 218 (1985)

130. W.M. Durham, J.O. Kessler, R. Stocker, Science 323, 1067 (2009)

131. T.J. Pedley, J.O. Kessler, Annu. Rev. Fluid Mech. 24, 313 (1992)

132. F.P. Bretherton, Rothschild, Proc. R. Soc. B 153, 490 (1961)

133. V. Kantsler, J. Dunkel, M. Blayney, R.E. Goldstein, eLife 3, e02403 (2014)

134. H. Winet, G.S. Bernstein, J. Head, J. Reprod. Fertil. 70, 511 (1984)

135. T. Kaya, H. Koser, Biophys. J. 102, 1514 (2012)

136. H. Marcos, T. Fu, Powers, R. Stocker, Proc. Natl. Acad. Sci. USA 109, 4780 (2012)

137. G.B. Jeffery, Proc. R. Soc. Lond. A 102, 161 (1922)

138. C. Torney, Z. Neufeld, Phys. Rev. Lett. 99, 078101 (2007)

139. N. Khurana, J. Blawzdziewicz, N. Ouellette, Phys. Rev. Lett. 106, 198104 (2011)

140. C. Zhan, G. Sardina, E. Lushi, L. Brandt, J. Fluid Mech. 739, 22 (2014)

141. B. ten Hagen, R. Wittkowski, H. Löwen, Phys. Rev. E 84, 031105 (2011)

142. A. Zöttl, H. Stark, Phys. Rev. Lett. 108, 218104 (2012)

143. A. Zöttl, H. Stark, Eur. Phys. J. E 36, 4 (2013)

144. M. Tournus, A. Kirshtein, L.V. Berlyand, I.S. Aranson, J. R. Soc. Interface 12, $20140904(2014)$

145. S. O’Malley, M.A. Bees, Bull. Math. Biol. 74, 232 (2012)

146. A. Chengala, M. Hondzo, J. Sheng, Phys. Rev. E 87, 52704 (2013)

147. S. Rafaï, L. Jibuti, P. Peyla, Phys. Rev. Lett. 104, 98102 (2010)

148. R.N. Bearon, A.L. Hazel, J. Fluid Mech. 771, R3 (2015)

149. M.T. Barry, R. Rusconi, J.S. Guasto, R. Stocker, J. R. Soc. Interface 12, 20150791 (2015) 